\title{
PRP: Product Rich in Placebo?
}

\author{
Giuseppe Filardo $^{1} \cdot$ Elizaveta Kon $^{1}$
}

Published online: 21 September 2015

(C) European Society of Sports Traumatology, Knee Surgery, Arthroscopy (ESSKA) 2015

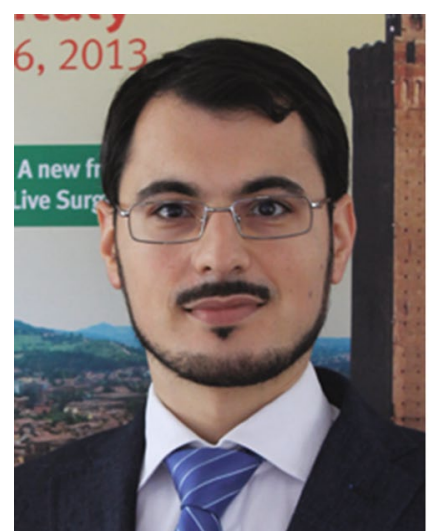

Giuseppe Filardo

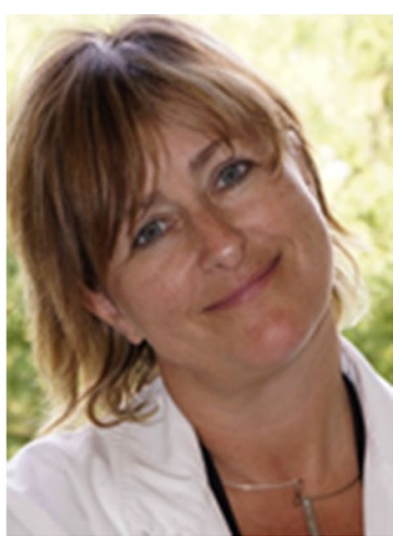

Elizaveta Kon
The search for a minimally invasive solution to improve the status of a pathologic joint surface and allow a fast return to full activity is highly desirable. In this landscape, a novel promising injective treatment is platelet-rich plasma (PRP), a blood derivative characterized by a higher platelet concentration than that of whole blood. When activated, platelets release a group of bioactive molecules that ultimately promote cellular recruitment, growth, and morphogenesis, and modulate inflammation as well [7]. Therefore, PRP is an appealing biological approach to favour the healing of tissues, such as cartilage, otherwise doomed by a low healing potential.

This led to the wide use of PRP, which shows promise to address joint degeneration processes in a minimally

Giuseppe Filardo

g.filardo@biomec.ior.it

1 Biomechanics Laboratory - II Clinic, Rizzoli Orthopaedic Institute, Via di Barbiano 1/10, 40136 Bologna, Italy invasive manner. In fact, one of the emerging uses of PRP treatment is its injective application for cartilage degeneration and osteoarthritis (OA), as shown by an increasing number of papers published on this topic over time [5]. Preclinical evidence supports PRP intra-articular (i.a.) use, targeting not only cartilage but rather promoting an overall favourable environment for joint tissues healing, with effects documented also at other levels, such as synovial and meniscal tissues [5].

The last decade has witnessed an increase in the popularity of PRP, with a peak in enthusiasm which has led to much talk and advertising about this biological treatment approach. However, this has been inadequately supported by a series of methodologically poor reports mainly indicating the safety and some preliminary promising findings. Nevertheless, the most recent literature presents a more favourable trend to show the real potential of PRP, with both an increase in preclinical studies to understand its mechanism of action and a decrease in low-level clinical reports but also an increase in the ratio of higher level trials.

Recent randomized controlled trials (RCTs) have shown overall support in favour of PRP i.a. injections, which has been shown to be superior than saline injections and also to a more traditional treatment, viscosupplementation [5, 6].

Nonetheless, the real potential of PRP for the treatment of knee degeneration is far from proven. In fact, the largest available double-blind randomized controlled trial comparing PRP and hyaluronic acid (HA) injections was not able to demonstrate any difference in the several subjective and objective outcome measures prospectively documented in 192 patients for up to 12-month follow-up [4]. It can be argued that the platelet concentrate used, its dose, timing, and modality of application may have influenced the outcome, thus explaining the conflicting results with other 
trials. It is also likely that many aspects such as cellularity, activation modality, better patient and disease indication need to be further explored to improve the potential of this biological treatment and that future developments will optimize PRP results and lead to a better outcome.

However, besides these common considerations which are mainly focused on product variability, another interesting observation may be made about the general concept of "platelet-derived growth factor" injections. The lack of difference in the latter study was not due to a lack of PRP effect, which was similar to previous studies in terms of improvement and duration, but to the different response of the patients to viscosupplementation. In fact, whereas in a previous un-blinded comparison HA presented a lower effect, drastically dropping at 6 months [8], when patients were blinded (and aware of the possibility of receiving "growth factors"), viscosupplementation offered both higher and longer lasting results, overlapping to those of PRP [4], thus supporting a significant placebo effect.

Research on "the powerful placebo" started in 1955 with a provocative article by Beecher [1] ascribing $35 \%$ of the clinical benefit to this effect, and over the last 60 years effects have been documented on all subjective outcomes (not just patient-centred, such as pain, stiffness, selfreported function, but also observer-centred), whereas the placebo has been seen as ineffective on most objective outcomes [9]. Thus, the value of the placebo effect is significant mainly for patients' symptoms and distress, which are the principal treatment targets in patients with OA, a condition where the pathology does not always correlate with symptoms [2], and may account for a large part of the documented PRP improvement.

PRP actually meets the requirements of an ideal placebo. Response to a placebo is irrespective of IQ [3], thus possibly affecting all patients, and the magnitude of the effect is determined largely by what patients are told about their treatment and by their expectations [2], which are considerably high when dealing with the fashionable "growth factor" PRP therapy. Besides response expectancy, branding and cost (treatment perceived as "high class"), novelty of the treatment, and route of delivery (needling is one of the most powerful, especially when performed repeatedly in injection cycles) [3] further support the marked components of the placebo effect in the overall improvement perceived by patients undergoing PRP i.a. injections.

PRP is a promising innovative approach, and increasing research efforts will provide a better understanding about its action mechanism, as well as the most suitable procedure to target specific patients and disease phases, which would probably optimize PRP results in the clinical practice. Nonetheless, it is important to remember the marked psychological effects related to this new and fashionable treatment, and future studies will need to couple the commonly used subjective scores with some objective outcome measures, to better define the placebo component of PRP and understand the real potential of this biological approach for the treatment of cartilage degeneration and knee OA.

\section{References}

1. Beecher HK (1955) The powerful placebo. J Am Med Assoc 159(17):1602-1606

2. Dieppe PA, Lohmander LS (2005) Pathogenesis and management of pain in osteoarthritis. Lancet 365(9463):965-973

3. Doherty M, Dieppe P (2009) The "placebo" response in osteoarthritis and its implications for clinical practice. Osteoarthritis Cartilage 17(10):1255-1262

4. Filardo G, Di Matteo B, Di Martino A, Merli ML, Cenacchi A, Fornasari P, Marcacci M, Kon E (2015) Platelet-rich plasma intra-articular knee injections show no superiority versus viscosupplementation: a randomized controlled trial. Am J Sports Med 43(7):1575-1582

5. Filardo G, Kon E, Roffi A, Di Matteo B, Merli ML, Marcacci M (2015) Platelet-rich plasma: why intra-articular? A systematic review of preclinical studies and clinical evidence on PRP for joint degeneration. Knee Surg Sports Traumatol Arthrosc 23(9):2459-2474

6. Görmeli G, Görmeli CA, Ataoglu B, Çolak C, Aslantürk O, Ertem K (2015) Multiple PRP injections are more effective than single injections and hyaluronic acid in knees with early osteoarthritis: a randomized, double-blind, placebo-controlled trial. Knee Surg Sports Traumatol Arthrosc. doi:10.1007/ s00167-015-3705-6

7. Kon E, Filardo G, Di Martino A, Marcacci M (2011) Plateletrich plasma (PRP) to treat sports injuries: evidence to support its use. Knee Surg Sports Traumatol Arthrosc 19(4):516-527

8. Kon E, Mandelbaum B, Buda R, Filardo G, Delcogliano M, Timoncini A, Fornasari PM, Giannini S, Marcacci M (2011) Platelet-rich plasma intra-articular injection versus hyaluronic acid viscosupplementation as treatments for cartilage pathology: from early degeneration to osteoarthritis. Arthroscopy 27(11):1490-1501

9. Zhang W, Robertson J, Jones AC, Dieppe PA, Doherty M (2008) The placebo effect and its determinants in osteoarthritis: meta-analysis of randomised controlled trials. Ann Rheum Dis 67(12):1716-1723 